\title{
Rural Physician-Pharmacist Collaborative Practice Agreements Managing Patients in Supportive Living and Assisted Living Memory Care Facilities
}

\author{
Mattie Haas, PharmD, MPA, BCGP1; LuAnn Haas, BSPh ${ }^{1}$; Kristine Knoke, BS, RN, BSN ${ }^{1}$; Michael Andreski, BSPh, MBA, PhD
}

${ }^{1}$ Nauvoo Pharmacy; Nauvoo, IL; ${ }^{2}$ Drake University College of Pharmacy and Health Sciences; Des Moines, IA

\begin{abstract}
Setting: Supportive living and assisted living memory care facilities in a rural West-Central Illinois county.

Objectives: 1) Evaluate the impact of active pharmacist participation on patient care for residents living in supportive and assisted living facilities, 2) demonstrate feasibility and financial sustainability of rural community pharmacists providing disease state management services, 3) create processes for best practice to expand the clinical role of the community pharmacist

Design: Case study.

Interventions: Participating residents received disease state management services provided by a community pharmacist as outlined through collaborative practice agreements with local physicians. The disease states managed included hypertension, hyperlipidemia, diabetes mellitus, and warfarin anticoagulation therapy. The pharmacist completed an initial chart review, initial face-to-face visit, subsequent monthly chart reviews, and monthly face-to-face visits with each resident.

Results: During the 6-month period of community pharmacist management, 86 face-to-face visits were completed to deliver a median of 5 visits per resident. The pharmacist identified 23 drug therapy problems with recommended solutions communicated to the resident's primary care provider. Providers accepted 19 of these recommendations, reflecting an $82.6 \%$ acceptance rate.

Conclusions: Community pharmacists can feasibly implement enhanced clinical services to assist with disease state management of supportive living and assisted living residents in collaboration with physicians. Pharmacists can provide clinical assessment, education and effective communication to optimize medication management and utilization.
\end{abstract}

Keywords: Collaborative practice agreement (CPA), senior living, rural, community pharmacy, disease state management

\section{Introduction}

The population of persons 65 years of age or older in the United States is expected to reach 83.7 million people by the year 2050. This is due to aging of the baby boomer generation and increasing life expectancy. ${ }^{1}$ As people age they are at higher risk of developing chronic disease, having multiple medications to treat the chronic disease, and needing advanced levels of coordinated care to maintain functionality. ${ }^{2}$ In addition to medication concerns, physical and cognitive impairments lead to varying levels of functionality of which some people can manage in their own home while others require various levels of skilled care associated with assisted living facilities (ALF) or long-term skilled nursing facilities. Assisted living facilities are viewed as a more residential-like and less institutionalized setting that does not have an overly regulated, inflexible environment as compared with a nursing home. ${ }^{3}$ Regulation and licensure for ALFs differs in rigidity and depth from state to state while skilled nursing home facilities are largely regulated at the federal level through Centers for Medicare and Medicaid Services (CMS). ${ }^{3}$

Corresponding author:

Mattie Ann Haas, PharmD, MPA, BCGP

Nauvoo Pharmacy; Nauvoo, IL

Email: mattieahaas@gmail.com
Residential care/assisted living facilities were rapidly growing in the United States in the early 2000s as an alternative to longterm skilled nursing facilities, to create an opportunity for higher functioning older adults to retain a level of independence. For less frail older adults, ALFs are a more appropriate level of care and use of resources. ${ }^{3}$ Consultant pharmacists have had a regulatory presence in the long-term skilled nursing setting as mandated by CMS performing drug regimen reviews (DRR) for each resident at least once a month, evaluating for unnecessary drugs, and assisting with the development and implementation of a care plan for each resident within 48 hours of admission; among other tasks. ${ }^{4}$ Assisted living facilities have not had this mandatory pharmacist oversight.

In 1991, the Beers Criteria outlining potentially inappropriate medication use in older adults was published and an emphasis to change medication regimens in persons 65 years of age and older to a safer or more effective alternative began. ${ }^{5}$ Research from the early 2000s reported a propensity to undertreat conditions and under prescribe medications to residents living in assisted living facilities in order to preserve independence and limit prescription overuse, adverse drug events, and drugdrug interactions. ${ }^{6}$ However in the last decade, research regarding residential care/assisted living has been lacking. The research available states the primary interventions are deprescribing of potentially inappropriate medications and medication management. ${ }^{6}$ The lack of information of 
pharmacists' impact on helping patients achieve therapeutic goals through collaborative practice agreements with rural physicians to perform disease state management in the assisted living and supportive living settings is the rationale for this project.

The beneficial involvement and accessibility of pharmacists as a member of the interdisciplinary healthcare team has been well documented in several settings including health systems and ambulatory care clinics. ${ }^{7-8}$ However, the adoption of clinically focused outpatient pharmacy services among the aging population within community settings has been much slower. Access to healthcare in rural areas for persons living in supportive living or assisted living facilities is suboptimal due to physician shortages and a relative lack of geriatric services. ${ }^{9-}$

${ }^{10}$ Literature suggests independence and quality of life, among other factors, have a positive correlation with the subjective perception of wellbeing among the aging. ${ }^{11}$ Adding the most accessible healthcare professional, a pharmacist, working in concert with the healthcare team to provide comprehensive reviews on medications and treatment care plans, can empower the aging patient to take responsible control of his or her health status. ${ }^{7}$ This in turn may allow the patient to remain at home or in a home-like setting (i.e., supportive living or assisted living facility) longer.

Pharmacists receive extensive training throughout their clinical curriculum to become effective communicators to both physicians and licensed nursing staff through the use of relevant clinical language. They also have the ability to counsel patients using patient-friendly language and to act as a liaison between the patient and healthcare providers. Pharmacists have the education and skills to perform disease state management services using evidence-based medicine published guidelines. This foundation of pharmaceutical care is synergistically realized when implemented within a collaborative practice agreement with a provider. Because rural medically underserved areas have provider shortages and limited geriatric services, the freeing up of providers to care for more complex conditions or acute health changes by pharmacists performing disease state management services is monumental.

The geriatric-focused pharmacist uses comprehensive medication expertise along with the physiologic knowledge of the aging body to help preserve functionality and quality of life for the aging person. With this in mind, pharmacist involvement may decrease medication related adverse reactions, prevent overmedication, lessen the use of potentially inappropriate medications, and recommend therapeutic alternatives for increased efficacy. Other aspects pharmacists can positively impact include enhancing cost containment as related to medication utilization, providing point-of-care testing, offering interpretation of objective results, and assisting with monitoring and follow-through of the existing care plan.

\section{Setting}

This study took place in a rural, socially and politically conservative, predominantly white community in a WestCentral Illinois county with a population of 18,500 . The county is primarily made up of large corn and soybean farms and is sparsely populated. The target population for the study consisted of a maximum of 62 residents living in Hancock Village Senior Services, which is comprised of two supportive living facilities (Hickory Grove: North and South) and two assisted living memory care facilities (Maple Grove: Red and Silver). Hancock Village was consistently $90-95 \%$ filled to capacity throughout the durations of the study. At the time of this study's initiation (2016), the Hancock Village campus was Illinois's first supportive living and assisted living memory care facility, constructed with funding from a grant from the United States Department of Agriculture. Hancock Village Senior Services is an affiliate of the local health system comprised of Memorial Hospital and Memorial Medical Clinics.

\section{Hickory Grove - Supportive Living}

Both Hickory South and Hickory North buildings each house approximately 20 residents. Licensed nursing staff is available during the day Monday through Friday and universal workers are present 24 hours daily in all facilities. A universal worker is trained to care for basic hygiene, perform basic housekeeping tasks, and provide medication or treatment plan reminders to the resident. However, they are not licensed or trained to handle medications. The residents living in Hickory Grove must be able to administer their own medications, which are kept in an accessible location in the apartment. Residents can either choose to manage their medications without assistance (i.e. keep medications in vials and take as prescribed) or they may request to have universal worker or nursing assistance (i.e. set up medications in planners or receive administration reminders).

\section{Maple Grove - Assisted Living Memory Care}

Both Red Maple and Silver Maple buildings each house approximately 9 residents. Licensed nursing staff is available during the day Monday through Friday and universal workers are present $\mathbf{2 4}$ hours daily in all facilities. The residents residing in Maple Grove have varying levels of memory impairment which requires routine licensed nursing oversight: such as evaluating any changes in health status, communicating health-related concerns to physicians, and ensuring resident attendance at scheduled physician appointments. Maple Grove residents must be able to administer their own medications, which are secured in a locked drawer in their own apartment. At the appropriate administration time, the resident receives a medication reminder by a universal worker who unlocks the drawer for the resident to take his or her medication. 


\section{Practice Description}

Gaps in care associated with medication utilization were identified at Hancock Village by the registered nurse on the research team. These gaps in care were primarily related to suboptimal continuity of care or siloed care: residents using multiple pharmacies, residents using multiple physicians/specialists, residents going 6-12 months in between doctor appointments, etc. Two of the major observations were delays in warfarin regimen adjustments after INR blood work and delays in ordering changes after providers adjusted medications at an office visit. When she observed these unnecessary delays regarding medication utilization, she brought it to the attention of the facility nursing staff and community pharmacists to problem solve for more efficient options.

Two community pharmacists from Nauvoo Pharmacy used the available opportunity for pharmacists' to actively manage medications through collaborative practice agreements as the solution to improve continuity of care and provide more effective follow-through relating to medication changes. Nauvoo Pharmacy is an independent, community pharmacy that serves the study area and has the vision to clinically integrate with the entire healthcare team. There were two community pharmacists, a registered nurse, and a professor of pharmacy on the research team. Of the two community pharmacists from Nauvoo Pharmacy, one primarily conducted the on-site visits with residents and made clinical recommendations ("clinical pharmacist") while the other handled the dispensing of medications and logistical needs of the project ("dispensing pharmacist"). The registered nurse performed the state-mandated quarterly assessments for every resident living at Hancock Village, but was not one of the facilities' main staff nurses. The professor of pharmacy assisted with study design, data analysis, and interpretation of results.

\section{Objectives}

The primary goal of the study was to determine the role and impact of community pharmacists' in performing clinical services in the supportive living and assisted living setting by assisting rural physicians and nurse practitioners in managing disease states through collaborative practice agreements, communicating more effectively with nursing staff, and empowering patients to achieve therapeutic outcomes in accordance with current national guidelines. The study objectives were 1) to evaluate the impact of active pharmacist participation on patient care for residents in supportive and assisted living settings, 2) to demonstrate the feasibility and sustainability of rural community pharmacists providing disease state management services, and 3) to create processes for best practice that can be used to expand the clinical role of the community pharmacist within the interdisciplinary team.
Methods

Study Design

The study design of this research was a case study.

\section{Study Population}

The target study population was approximately 60 persons residing at Hancock Village diagnosed with one or more of the following disease states: hypertension, hyperlipidemia, diabetes mellitus, and warfarin anticoagulation therapy. There was no inclusion or exclusion data based upon gender, race, or age.

\section{Participant Recruitment}

A packet was sent out to each resident, which included a letter of explanation of the study, a sample of the compliance packaging, and a biography sheet describing the researchers. A meeting at Nauvoo Pharmacy was held for the Hancock Village licensed nursing staff, collaborating physicians, and facility administrators to describe the anticipated workflow, demonstrate the new compliance packaging, and answer questions. The research nurse and clinical pharmacist attended a medical staff meeting at Memorial Medical Clinics to inform the entire medical staff of the program, goals, benefits, and to answer any questions. The research nurse and clinical pharmacist attended a full staff meeting at Hancock Village including universal workers, administrators, and other staff members to inform them of the new program. Open meetings explaining the goals, risks and benefits of the study, and answering questions or concerns for interested residents and family members were held about two weeks after the packets were sent out. These meetings were sparsely attended with only 4 study participants signing up. One-on-one recruiting was then employed during each resident's quarterly nursing assessment. This method was more successful with nine of the study participants gradually recruited over the last 4 months of the data collection phase. As stated previously, the research nurse completed the state-mandated quarterly assessments for each resident and continued to mention the purpose and benefits of the study and encourage participation.

\section{Data Collection}

Collaborative practice agreements (CPAs) were created and signed by all three participating rural physicians for hypertension, hyperlipidemia, diabetes mellitus and warfarin anticoagulation therapy before the data collection period began. All CPAs were created in accordance with current national guidelines for each disease state as established from evidence-based medicine (i.e. JNC 8, AHA/ACC, ADA, AACE/ACE, and CHEST) and outlines were adopted from Community Health Association of Spokane, Washington (CHAS). The CPAs gave the pharmacist the authority to make changes to medication therapy including initiation, adjustments, and discontinuations. Informed consent was obtained from each study participant or the appropriate patient representative before data collection began. 
Participants were managed by the pharmacist for multiple conditions covered within the scope of the CPAs. The study and all recruitment material were approved by the Drake University Institutional Review Board.

\section{Care Model Development}

The clinical community pharmacist was credentialed as a Board Certified Geriatric Pharmacist (BCGP). She completed an initial chart review to familiarize herself with each resident's past medical history, a current reconciled medication list, most recent laboratory results, allergies, current co-morbidities, and main health concerns. After the initial chart review, monthly chart reviews were performed that focused on changes in medications and health status. Each study participant was visited face-to-face initially and then monthly, or more often if medications were adjusted, to assess adherence, medication concerns, and perform objective tests such as blood pressure, heart rate, International Normalized Ratio (INR), etc., as required for disease state management. The clinical pharmacist was granted access to Memorial Medical's electronic medical record to obtain objective data. A read-only version of Dispill, the pharmacy software used for creating compliance packaging labels and supplying Medication Administration Records (MARs) to the nursing staff, was installed on Hancock Village nursing staff computers. This provided a real-time medication list for doctor appointments or emergency room visits and location for the nursing staff to view notes made by the pharmacists about medication adjustments. A Clinical Laboratory Improvement Amendments (CLIA) waiver was obtained for the purpose of point-of-care testing using the CoaguChek ${ }^{\circ}$ device for residents on warfarin anticoagulation therapy. The data collection period spanned six months from February 28 to August 31, 2017.

\section{Results}

A total of 15 of the approximate 60 residents residing at Hancock Village participated and completed the study. Eleven of the participants lived in Hickory Grove (supportive living) and four lived in Maple Grove (assisted living). Pharmacist interventions were tracked for the four disease states of hypertension, hyperlipidemia, diabetes mellitus, and warfarin anticoagulation therapy in relation to corresponding CPAs. Of the 15 participants, 14 were managed for hypertension, 9 for hyperlipidemia, 3 for diabetes mellitus, and 1 for warfarin anticoagulation therapy. Residents diagnosed with multiple comorbidities, inclusive of the CPAs, were managed for those conditions.

During the six-month period of community pharmacist management, 86 face-to-face visits were completed. A median of 5 visits per resident occurred during the six-month data collection phase. [Table 1]
Table 1.

\begin{tabular}{|l|l|l|}
\hline Month & $\begin{array}{l}\text { Residents } \\
\text { Enrolled }\end{array}$ & $\begin{array}{l}\text { Pharmacist- } \\
\text { Resident Visits }\end{array}$ \\
\hline February & 4 & 4 \\
\hline March & 6 & 6 \\
\hline April & 6 & 6 \\
\hline May & 10 & 12 \\
\hline June & 13 & 14 \\
\hline July & 14 & 16 \\
\hline August & 15 & 28 \\
\hline Total & $\mathbf{1 5}$ & $\mathbf{8 6}$ \\
\hline
\end{tabular}

Twenty-three drug therapy problems were identified with recommended solutions communicated to the residents' primary care providers. Nineteen of these pharmacist-initiated recommendations were accepted by providers, which reflected an $82.6 \%$ acceptance rate. The breakdown of drug therapy problems identified with accepted and rejected recommendations is detailed in Appendix A.

Previous pneumococcal (Prevnar13 and Pneumovax) and shingles (Zostavax) vaccination history was assessed during the initial chart review. Seven Prevnar13 vaccines, 4 Zostavax vaccines, and 11 High-Dose Fluzone vaccines were administered during the study period. No Pneumovax vaccines were administered during this study due to the Centers for Disease Control and Prevention (CDC) recommended 12month separation period between Prevnar13 and Pneumovax.

\section{Impact on patient care}

The greatest impact of the community pharmacist during this study was improved lines of communication with providers, nursing staff, and patients and optimization of medication regimens in accordance with CPAs. Through repeated interactions during the monthly face-to-face visits, not only were desired disease state outcomes achieved or maintained (A1c $<7 \%, \quad B P<140 / 80$, etc.), but trusting and caring relationships developed between the resident and pharmacist. The community pharmacists were able to relay patient concerns to the provider and address patient health-related issues that may never have been brought to the provider's attention. Each resident needed to be capable of selfadministering all medications, which proved to be difficult with complex medication regimens or technique administration challenges. Residents would share or be prompted to share if they were experiencing challenges with medication administration such as having to take large doses of medication at any given administration time or experiencing an adverse effect such as an antidepressant causing daytime drowsiness.

\section{Individual Patient Success Stories}

One study participant was managed for warfarin anticoagulation therapy. She agreed to participate in the study with two months remaining in the data collection phase. The researchers were only able to actively manage her warfarin 
therapy in the last month of the study due to an unplanned visit to the emergency room in the second-to-last month of data collection. Her INR was evaluated using the CoaguChek', a point-of-care testing device. The patient said she was thrilled the results were available within seconds and that it took a finger prick instead of a venous puncture.

A study participant who was cognitively difficult to reach, but had an outstanding outcome was a resident of Hickory Grove. He managed his own medications and would only ask for assistance when calling in a refill. He stopped taking his levothyroxine because he believed the iodine in his multivitamin was doing the same job. His doctor gradually kept increasing his levothyroxine dose as his Thyroid Stimulating Hormone (TSH) continued to rise. Upon noticing the uptrend and realizing the levothyroxine had not been refilled since the last dose increase, the pharmacist had a conversation with the resident about adherence. It was at this time the misbelief about the iodine was discovered. After speaking with the resident about how levothyroxine works and why it is important to take every day, he agreed to allow the pharmacy to set up his medications in the compliance packaging. His next laboratory tests after this intervention yielded a TSH level within normal limits.

Several of the residents stated they appreciated knowing someone else cared about them and their health. On-site nursing staff was not present to administer medications and the universal workers are not trained to handle medication. The community pharmacists had a two-fold impact on the health of the residents. They were able to provide disease state management services utilizing the protocols outlined in the CPAs as evidenced by the identification and resolution of drug therapy problems. The community pharmacists were also able to provide a level of emotional support that allowed the resident to feel like an engaged participant in his or her own health care.

\section{Improved Communication}

Improved quality of communication with the nursing staff at Hancock Village was a beneficial outcome of the study. As a trusting work relationship developed between the nursing staff and community pharmacists, the nursing staff began sharing criticisms and feedback of the medication dispensing processes and the community pharmacists were able to provide solutions for improved nursing workflow. This was primarily achieved through consistent telephone calls, emails, faxes, and sharing electronic access to Nauvoo Pharmacy's packaging software with the nursing staff. Granting community pharmacists access to health system electronic medical records was considered an accomplishment by the research team and Nauvoo Pharmacy was able to reciprocate electronic access for the nursing staff at Hancock Village.
Methods of Feasibility and Sustainability

Although a sustainable payment model was not implemented during the duration of the study, potential methods of financial sustainability were recognized. The first method was to approach the health system with the idea of hiring the community pharmacist as an employee of the facility. This implies every resident would receive services provided by the community pharmacist and the pharmacist would be available to meet other needs the facility may have. The proposed cost of the pharmacist would be included in each resident's monthly housing expense and divided equally. This method seems to be the most attainable and simplistic in the short term because it does not involve figuring out how to bill for a pharmacist's clinical services through the health system's billing software. This is a challenge because pharmacists do not have independent provider status and most billing systems do not have an existing avenue to bill for pharmacist's services.

Including a clinically focused pharmacist on the facility treatment team can be of economical benefit to the facility. There are no other assisted living or supportive living facilities within a 50-mile radius that utilize the service of a clinical pharmacist (2017). Having a pharmacist involved in medication therapy management would be a way to differentiate the facility from others in order to achieve a higher or to-capacity occupancy rate. Conceptually, it is also anticipated that with the increased clinical pharmacist oversight, the resident would require fewer unplanned visits to the doctor's office and fewer emergency room visits. More medically stable residents may require fewer resources and care from staff, and the facility may experience less turnover of residents to skilled nursing facilities.

Another potential source of financial sustainability was billing for medication management services using incident-to billing codes through the local health system affiliated with Hancock Village Senior Services and providing the health system with a portion of the reimbursement. This method was not realized yet due to logistical and regulatory challenges associated with creating an avenue to bill for pharmacist services.

Another method considered for obtaining financial sustainability was joining the Illinois Community Pharmacy Enhanced Network (I-CPEN) with support from the national Community Pharmacy Enhanced Service Network (CPESN USA). As defined by CPESN USA, "pharmacies providing Enhanced Services that transcend conventional requirements of an outpatient pharmacy program contract and focus on improving clinical and global patient outcomes."12 The vision of I-CPEN is a network of community-focused pharmacies around the state that provide an elevated set of pharmaceutical care services to patients in collaboration with healthcare providers, health systems, Accountable Care Organizations (ACO), payers and pharmaceutical industry via a single contracting entity to document and achieve improved health outcomes..$^{12}$ At the conclusion of the study, I-CPEN was 
just laying groundwork to establish the foundation of the network. This is a promising alliance moving forward as the developed network and high-performing community pharmacies unite together.

\section{Processes for Best Practice}

Through the course of the data collection period, a best practice model for a community pharmacist providing services to residents residing in an assisted living and supportive living setting was identified. [Appendix B] An ever-improving system was developed and outlined for medication packaging and delivery, communication pathways to the providers and licensed nursing staff, and efficient workflow processes for the pharmacist to complete tasks. The care model emphasizes the importance of establishing mutually respected working relationships between the pharmacists, providers, facility administers and staff before the service begins. Outlining a quality assurance standard to continually improve workflow, provide clinical documentation, ensure sustainability, and demonstrate accountability illustrates the value a pharmacistinitiated service can provide to the interprofessional care team.

\section{Discussion}

Challenges

There were several challenges to initiating clinically focused pharmacist services at Hancock Village. At the time of study initiation, Nauvoo Pharmacy was not providing medications for the residents of Hancock Village. Study participants had to have their medication dispensing transferred to Nauvoo Pharmacy, which took varying amounts of time and organization including obtaining new or transferred prescriptions and completing a comprehensive reconciled medication list. The decision to have Nauvoo Pharmacy exclusively fill the study participants' medications provided control in communication and medication management rather than working as a third party in-between the dispensing pharmacy and facility. Many of the residents were hesitant of this change due to the fear of the unknown that comes along with change or because of hometown loyalty to the current dispensing pharmacy. The prior dispensing pharmacy made some efforts to retain the residents, which also contributed to this hesitancy. Some residents took several months to decide to participate in the study. In fact, 9 of the 15 study participants only decided to participate gradually over the last 4 months of the data collection phase.

Another challenge was working with prescribers who were not familiar with working with pharmacists as medication therapy managers. The lack of collaboration resulted in a significant delay in reaching a desired therapeutic outcome in at least one patient.

The physician champion and Hancock Village medical director left the area midway through the data collection period. This resulted in a wider gap between the supportive physicians and resistant nurse practitioners making it difficult to continue to recruit study participants.

During the study period, the local health system also began transitioning to a new health information system that affected the connected physicians' offices and the hospital where many of the residents of Hancock Village received the majority of their care. Although supportive conversations to facilitate project progress with administrators occurred, getting followthrough from them was difficult due to a number of changes within the health system.

A great deal of education was necessary to get the facility nursing staff to facilitate an effective working relationship with Nauvoo Pharmacy. The amount of education needed was underestimated in the beginning of the project. The study required establishing more effective communication methods, new medication dispensing processes providing education to the nursing staff, and training the nursing staff on the scope of services and procedures to deliver accepted services. As a result of the study, improved relationships with the facility nursing staff were evident from an increase in volume of communication, directed medication-related questions, and a willingness to provide constructive feedback. The improved communication also made the process of procuring a reconciled medication list easier and less time consuming. This enhanced working relationship with nursing was made possible by keeping them consistently and thoroughly informed and following through with tasks as soon as possible.

Another challenge was inconsistent timing and availability of both the clinical community pharmacist and licensed nursing staff at the facilities due to ever-changing schedules. It took rearranging schedules and consistent, prospective communication primarily via email to find available days and consecutive hours to coordinate meeting in person with nursing staff.

Recruitment for project participation proved to be challenging. This effort was underestimated, in the beginning, due to the involved process of identifying who was the decision maker: the resident, family member, or other named resident representative. One-on-one meetings with the resident or resident's family/power of attorney proved to be the most effective form of recruitment. Moving forward, this challenge could be circumvented with clinical pharmacist services included in the facility's services available to all residents.

\begin{abstract}
Limitations
The small sample size of 15 residents was a limitation. Having a small sample size may mean that the results of the study may not represent the effects if the study was performed in a larger population or a population that is more socioeconomically diverse. Another limitation resulting from the small sample size was limitation to a case study design instead of a full quantitative analysis. Reasons for the small sample size may
\end{abstract}


have been the recruitment strategy, attitude of resistance to change, or incomplete understanding of what benefits might have been realized from participation. The reluctance of nurse practitioners to accept collaboration with the community pharmacists may have kept residents from being willing participants in the study. Nurse practitioners from the local health care system provided the majority of the primary health care for the residents of Hancock Village. A possible way to address this limitation in the future could be to focus on establishing a better working relationship with providers during the project initiation phase.

\section{Conclusion}

Community pharmacists actively managing medications through collaborative practice agreements can positively impact supportive living and assisted living memory care residents' overall quality of life, provide clinical value to rural providers, and reduce healthcare costs. Pharmacists can provide clinical assessment, medication and disease state education, along with effective communication to optimize medication management and utilization. Incorporating a community pharmacist as a member of the rural healthcare team for residents in a supportive living or assisted living facility is feasible. Securing a sustainable financial model in any start-up pharmacy service is still a challenge due to pharmacists not having provider status to bill independently for clinical services. Further areas of study to expand the utility of this geriatric-focused medication management process could extend to the aging population living independently in their own homes.

The combined force and dedication of active community pharmacists providing enhanced services in managing medication therapy for all patients would give depth of objective data and an additional perspective to the interprofessional healthcare team to help patients achieve health-related goals.

The authors are willing to share collaborative practice agreements and standing orders with anyone interested in obtaining copies.

Acknowledgements: We would like to thank the administrators and nursing staff at Hancock Village Senior Services, participating physicians and nurse practitioners, and the Community Pharmacy Foundation for the opportunity to serve our rural community.

Conflicts of Interest: None

Funding: This work was supported by the Community Pharmacy Foundation [grant \#71325, 2017]

Treatment of Human Subjects: IRB review/approval required and obtained through Drake University Institutional Review Board.

\section{References}

1. Ortman JM, Velkoff VA, Hogan $\mathrm{H}$. An aging nation: the older population in the United States. Current Population Reports P25-1140. Washington, DC: US Census Bureau; 2014

2. Swanoski M, Little M, St. Hill C et al. Potentially Inappropriate Medication Prescribing in U.S. Older Adults with Selected Chronic Conditions. The Consultant Pharmacist 2017; 32:525-34

3. Golant SM. Do Impried Older Persons With Health Care Needs Occupy U.S. Assisted Living Facilities? An Analysis of Six National Studies. Journal of Gerontology 2004; 59B(2):568-579

4. Final Rule: Requirements for Participation Mega Rule. American Society of Consultant Pharmacists. https://cdn.ymaws.com/www.ascp.com/resource/re smgr/docs/issues/megarule/mega-rule-summarychart.pdf. 26 Apr 2019

5. American Geriatrics Society 2015 Beers Criteria Update Expert Panel. American Geriatrics Society 2015 Updated Beers Criteria for Potentially Inappropriate Medicate Use in Older Adults. Journal of the American Geriatrics Society. 2015; 63:22272246

6. Sloane PD, Gruber-Baldini AL, Zimmerman S, et al. Medication Undertreatment in Assisted Living Settings. Archives of Internal Medicine American Medical Association. 2004; 164:2031-2037

7. Twigg GA, Mostsko J, Sherr J, El-Baff S. Interprofessional Approach to Increase Billable CareEvents in a Rural Community. Innovations in Pharmacy Journal. 2017;8(1): Article 14. http://pubs.lib.umn.edu/innovations/vol8/iss1/14

8. Steele KM, Ruisinger JF, Bates J, et al. Home-Based Comprehensive Medication Reviews: Pharmacist's Impact on Drug Therapy Problems in Geriatric Patients. The Consultant Pharmacist 2016; 31:598605

9. Galambos CM. Health care disparities among rural populations: a neglected frontier. Health and Social Work, vol. 30, no. 3, Aug 2005. BiomedSearch.com, www.biomedsearch.com/article/Health-caredisparities-among-rural/135466735.html

10. Rosenthal TC, Fox C. Access to Health Care for the Rural Elderly. JAMA. 2000;284(16):2034-36. doi:10.1001/jama.284.16.2034. 5 Jan 2018

11. Binstock RH, George LK, Cutler SJ et al. Handbook of Aging and the Social Sciences. Burlington: Elsevier Inc, 2006. www.books.elsevier.com. 18 Nov 2017

12. Illinois Community Pharmacy Enhanced Network. https://www.i-cpen.org. Web. 5 Jan 2018

13. Health Resources \& Services Administration Data Warehouse. U.S. Department of Health and Human Services.

https://datawarehouse.hrsa.gov/tools/analyzers/mu afind.aspx. Web. 5 Jan 2018 
Appendix A

Description of Pharmacist-Initiated Interventions

\begin{tabular}{|c|c|c|c|}
\hline $\begin{array}{l}\text { Drug Therapy } \\
\text { Problem (DTP) }\end{array}$ & $\begin{array}{l}\text { Total DTPs } \\
\text { Identified }\end{array}$ & $\begin{array}{l}\text { Accepted (A)/ } \\
\text { Rejected (R) }\end{array}$ & Description of DTP \\
\hline $\begin{array}{l}\text { Unnecessary Drug } \\
\text { Therapy }\end{array}$ & 4 & $4 / 0$ & $\begin{array}{l}\text { 1. Discontinued therapeutic duplication (ranitidine and famotidine) - } \\
\text { A } \\
\text { 2. Removed Mucinex DM (tablets and liquid) q6hr prn and changed } \\
\text { to Mucinex BID - A } \\
\text { 3. Removed milk of magnesia daily prn - had never used and has } \\
\text { polyethylene glycol daily prn that she prefers to use - A } \\
\text { 4. Removed multiple vitamin duplications and kept patient on a } \\
\text { single multivitamin for women - A }\end{array}$ \\
\hline Wrong Drug & 4 & $3 / 1$ & $\begin{array}{l}\text { 1. Changed Namenda XR 28mg to Namenda } 10 \mathrm{mg} \text { BID for cost } \\
\text { savings - A } \\
\text { 2. Changed daily Vitamin D 1,000 units to once weekly Vitamin } \\
10,000 \text { units to minimize pill burden - A } \\
\text { 3. Patient on melatonin with no sleep improvement - } \\
\text { recommended to change to low-dose mirtazapine - R } \\
\text { 4. Patient fell while on liquid Benadryl and no sleep improvement - } \\
\text { recommended to change to low-dose mirtazapine - A }\end{array}$ \\
\hline Dose too low & 4 & $3 / 1$ & $\begin{array}{l}\text { 1. Increased lisinopril from } 5 \mathrm{mg} \text { to } 10 \mathrm{mg} \text { - A } \\
\text { 2. Increased lisinopril from } 10 \mathrm{mg} \text { to } 20 \mathrm{mg} \text { - A } \\
\text { 3. Increased lisinopril from } 20 \mathrm{mg} \text { to } 40 \mathrm{mg} \text { - A } \\
\text { 4. Recommended increase lisinopril from } 40 \mathrm{mg} \text { to } 40 \mathrm{mg} \text { BID - R }\end{array}$ \\
\hline Dose too high & 1 & $1 / 0$ & $\begin{array}{l}\text { 1. Reduced risperidone } 1 \mathrm{mg} \text { TID to risperidone } 0.5 \mathrm{mg} \text { TID due to } \\
\text { falls/confusion/ataxia - A }\end{array}$ \\
\hline $\begin{array}{l}\text { Adverse Drug } \\
\text { Reaction }\end{array}$ & 3 & $3 / 0$ & $\begin{array}{l}\text { 1. Changed administration time of citalopram to HS due to daytime } \\
\text { drowsiness - A } \\
\text { 2. Changed administration time of famotidine to HS due to potential } \\
\text { dementia-like side effects and reduced renal function - A } \\
\text { 3. Changed Actos to Januvia - patient experiencing moderately } \\
\text { significant lower extremity edema (likely due to comorbidity } \\
\text { heart failure - patient on high dose furosemide) - A }\end{array}$ \\
\hline $\begin{array}{l}\text { Needs additional } \\
\text { drug therapy }\end{array}$ & 3 & $1 / 2$ & $\begin{array}{l}\text { 1. Initiated lisinopril due to resistant hypertension and diabetes - A } \\
\text { 2. Recommended missing statin therapy on a diabetic patient - R } \\
\text { 3. Recommended initiating fish oil for elevated triglyceride level - R }\end{array}$ \\
\hline
\end{tabular}




\section{Appendix B \\ Assisted Living Memory Care and Supportive Living Best Practice Model}

Outline of a clinically focused pharmacist service to provide medication management as an included service to ALL residents living in Hancock Village

1) Establish mutually respected relationships with licensed nursing staff and administrators at facilities and share characteristics of clinical pharmacist service

2) Establish mutually respected relationships with area primary care providers (provider in-service meetings, one-on-one meetings with providers, etc.) and share characteristics of clinical pharmacist service

3) Create collaborative practice agreements (CPAs) in accordance with current national guidelines for each intended disease state as established from evidence-based medicine. Get CPAs signed by participating primary care providers

4) Provide education to residents on a one-on-one basis upon admission - have resident sign acknowledgement that this information has been reviewed and what he or she should expect

5) Admit resident

a. Procure medication list from family and doctor's office

b. Inspect medication vials when feasible (confirm medication strength, directions, prescribing doctor, and any other visual clues)

c. Complete medication reconciliation before medications lists are sent to pharmacy for dispensing (this cleans up the process moving forward)

d. Complete an initial chart review to familiarize with past medical history (History \& Physical), more recent laboratory results, allergies, current co-morbidities, and main health concerns

6) Meet with resident for assessment and evaluation of his or her current medication utilization (Initial meeting up to 45 minutes, and follow-up meetings 20-30 minutes)
a. Formulate a medication management care plan
b. Review plan with resident, licensed nursing staff, and primary provider
c. Send medication therapy management plan to dispensing pharmacy

7) Follow-up - monthly clinical pharmacist chart review to assess for changes in health status and medications and face-toface meeting with resident. In addition, quarterly face-to-face pharmacist and nursing physical assessments with resident/lab review/discussion would be coordinated with licensed nursing staff

a. Make further recommendations to medication management care plan as warranted

b. Update medication management care plan and inform nursing staff, providers, and dispensing pharmacy

i. Updates will be relayed to provider via electronic record

8) Continue the cycle

\section{Goals/Objectives}

1) Demonstrate need, feasibility and financial sustainability of clinical pharmacist service to manage disease states in partnership with rural primary care providers

2) Create process for best practice in the supportive living and assisted living setting to be used as a model for other like facilities

3) Evaluate the impact of active pharmacist participation on patient care

a. Track the number of doctor/emergency room visits

b. Track the number of acute hospital admissions/readmissions in local health system

c. Track turnover of resident apartments/empty apartments

d. Track ability of pharmacist to recognize early changes in health status and interventions made to prevent further deterioration

e. Track interventions made to maximize correct medication utilization (administration and technique)

f. Create opportunity for residents or resident representatives to provide comments on pharmacist services and evaluate value of feedback 


\section{System Analysis/Quality Assurance Program}

The Quality Assurance team will consist of the pharmacist, facility administrator, lead clinical nurse, medical director, and facility staff nurses. The team will meet at least annually and more often as needed for one hour.

1) Compile pharmacist interventions (identify proposed drug therapy problem recommendations and accepted vs. declined and the trend thereof)

2) Evaluate therapeutic response secondary to medication adjustment

3) Make process improvement recommendations as identified to outline best practice model (based on the results of the quality assurance meetings the process will be modified to meet the needs of the residents)

4) Ensure sustainability - track expenses (pharmacist time vs. revenue), be diligent in finding new methods to stay financially current 